This article was downloaded by: [B-on Consortium - 2007]

On: 25 August 2009

Access details: Access Details: [subscription number 908038079]

Publisher Taylor \& Francis

Informa Ltd Registered in England and Wales Registered Number: 1072954 Registered office: Mortimer House, 37-41 Mortimer Street, London W1T 3JH, UK

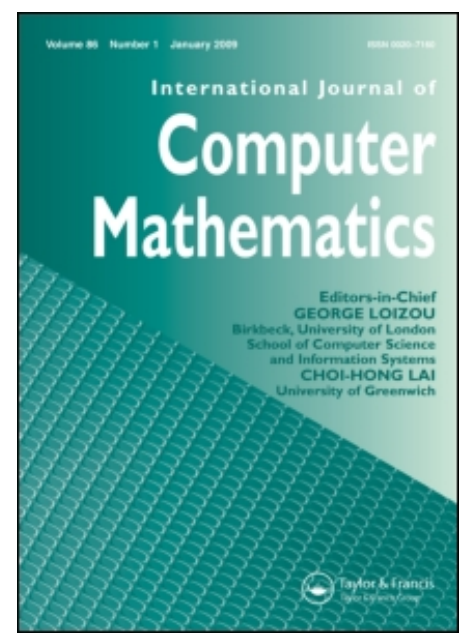

\title{
International Journal of Computer Mathematics
}

Publication details, including instructions for authors and subscription information: http://www.informaworld.com/smpp/title content=t713455451

\section{Lifting solutions of quasilinear convection-dominated problems}

\section{J. A. Ferreira a; A. P. Mouro a; P. Oliveira}

a CMUC, Department of Mathematics, University of Coimbra, Portugal

First Published on: 30 April 2009

To cite this Article Ferreira, J. A., Mouro, A. P. and Oliveira, P.(2009)'Lifting solutions of quasilinear convection-dominated problems', International Journal of Computer Mathematics,99999:1,

To link to this Article: DOI: $10.1080 / 00207160802385800$

URL: http://dx.doi.org/10.1080/00207160802385800

\section{PLEASE SCROLL DOWN FOR ARTICLE}

\footnotetext{
Full terms and conditions of use: http://www.informaworld.com/terms-and-conditions-of-access.pdf

This article may be used for research, teaching and private study purposes. Any substantial or systematic reproduction, re-distribution, re-selling, loan or sub-licensing, systematic supply or distribution in any form to anyone is expressly forbidden.

The publisher does not give any warranty express or implied or make any representation that the contents will be complete or accurate or up to date. The accuracy of any instructions, formulae and drug doses should be independently verified with primary sources. The publisher shall not be liable for any loss, actions, claims, proceedings, demand or costs or damages whatsoever or howsoever caused arising directly or indirectly in connection with or arising out of the use of this material.
} 


\title{
Lifting solutions of quasilinear convection-dominated problems
}

\author{
J.A. Ferreira*, A.P. Mouro and P. Oliveira \\ CMUC, Department of Mathematics, University of Coimbra, Portugal
}

(Received 02 November 2007; revised version received 04 March 2008; second revision received 19 April 2008; third revision received 23 May 2008; accepted 23 May 2008)

\begin{abstract}
In certain cases, quasilinear convection-diffusion-reaction equations range from parabolic to almost hyperbolic, depending on the ratio between convection and diffusion coefficients. From a numerical point of view, two main difficulties can arise related to the existence of layers and/or the non-smoothness of the coefficients of such equations. In this paper we study the steady-state solution of a convection-dominated problem. We present a new numerical method based on the idea of solving an associated modified problem, whose solution corresponds to a lifting of the solution of the initial problem. The method introduced here avoids an a priori knowledge of the layer(s) location and allows an efficient handling of the lack of smoothness of the coefficients. Numerical simulations that show the effectiveness of our approach are included.
\end{abstract}

Keywords: convection-dominated problem; non-uniform meshes; convergence

2000 AMS Subject Classifications: 35B45; 65L12; 65L20; L5L50; 655N50

\section{Introduction}

Quasilinear advection-diffusion-reaction equations of type

$$
u_{t}-\nabla \cdot(D(u) \nabla u)+b(u) \nabla u+c(u)=0, \quad(x, t) \in(0, T] \times \Omega,
$$

arise naturally in many mathematical processes such as flow in porous media petroleum reservoir and groundwater aquifer simulation $[1,3,6]$, and more generally in an extraordinary variety of chemical and biological phenomena [19]. In Equation (1), $\Omega$ represents a bounded set in $\mathbb{R}^{n}, \nabla u$ denotes the gradient of $u$ and

$$
\nabla \cdot(D(u) \nabla u)=\sum_{i=1}^{n} \frac{\partial}{\partial x_{i}}\left(D(u) \frac{\partial u}{\partial x_{i}}\right) .
$$

*Corresponding author. Email: ferreira@mat.uc.pt

ISSN 0020-7160 print/ISSN 1029-0265 online

(C) 2009 Taylor \& Francis

DOI: $10.1080 / 00207160802385800$

http://www.informaworld.com 
In certain cases the nonlinear convection-diffusion-reaction equation ranges from parabolic to almost hyperbolic, depending on the ratio between convection and diffusion coefficients.

From a numerical point of view, two main difficulties can arise in the solution of this kind of problems related to the existence of layer(s) and/or the non-smoothness of the coefficients. As far as the first difficulty is concerned, numerical methods defined on uniform grids suffer from severe non-physical oscillations or excessive numerical diffusion. These unphysical behaviours can be eliminated considering several approaches (see, for instance [7-10,14,16-18,22]. The numerical difficulties due to the lack of smoothness when Newton's method is used to solve the algebraic system resulting from the discretization are considered, for example, in $[2,4,11,12]$.

The aim of this paper is to present a new numerical method for solving the steady-state boundary value problem (BVP)

$$
\begin{gathered}
-D \Delta u+b(u) \nabla u+c(u)=0 \quad \text { in } \Omega=(0,1), \\
u(0)=A, \quad u(1)=B,
\end{gathered}
$$

with $B \neq A$, which handles efficiently the difficulties posed by the convection-dominated case.

The idea underlying our method is that to solve a problem with a mesh of high density in the layer(s) is equivalent to solving a modified equation with a more regular solution in an equidistant mesh. Roughly speaking, we define a mesh-generating function such that the initial BVP is equivalent to a modified problem, whose solution is a first-order polynomial. This corresponds to selecting a mesh with a density proportional to the gradient of $u$. In fact, let $u$ be the solution of (2), and let $x=g(\xi)$ be the mesh-generating function. If $\bar{u}(\xi):=u(g(\xi))$ we choose $g$ such that $\bar{u}(\xi)=(B-A) \xi+A$ is a solution of (2), and then

$$
\bar{u}_{\xi}(\xi)=u_{x}(g(\xi)) g_{\xi}(\xi)=(B-A),
$$

which corresponds to consider $g$ such that

$$
\frac{1}{g_{\xi}(\xi)}=\frac{u_{x}(g(\xi))}{B-A},
$$

where $1 / g_{\xi}(\xi)$ stands for a representation of the mesh density.

We represent the derivatives of $u$ and $g$ by $u_{x}$ and $g_{\xi}$, respectively. The second-order derivatives will be denoted by $u_{x^{2}}$ and $g_{\xi^{2}}$.

The change of variable considered transforms problem (2) into a differential equation - the mesh equation - where the dependent variable is $g$. A new method for solving this mesh problem is proposed, leading to an approximation $g_{k}\left(\xi_{i}\right)$ of $g\left(\xi_{i}\right)$. It can be proved that $u_{h}\left(x_{i}\right)=(B-$ $A) \xi_{i}+A$ is the solution of a non-standard finite-difference discretization of (2), defined on the non-equidistant mesh $\left\{g_{k}\left(\xi_{i}\right)\right\}$ computed with the proposed method.

The non-standard finite-difference discretization for $u_{h}$ is inconsistent in the computational grid $\left\{g_{k}\left(\xi_{i}\right)\right\}$, but it is consistent in the mesh defined by $\left\{g\left(\xi_{i}\right)\right\}$. Nevertheless we show that such finite-difference discretrization is convergent with respect to $\|\cdot\|_{\infty}$. The convergence properties of the finite-difference approximation of the mesh equation play a central role in the convergence analysis carried on for $u_{h}$.

The advantage of our approach is that the solution of only one equation - the mesh equation gives not only the localization of the layers but also the solution of (2) and allows an efficient handling of the lack of smoothness of the coefficients. An extension of the procedure for multidimensional domains of type $\Omega=\prod_{i=1}^{n}\left(a_{i}, b_{i}\right)$ is being addressed.

The paper is organized as follows. In Section 2 we define a mesh-density function and construct the mesh equation. In Section 3 a discretization of the mesh equation is studied, and we prove 
that the numerical solution computed in such a mesh is a solution of a non-standard, nonlinear, finite-difference method. The convergence of the finite-difference solution obtained with the nonstandard, nonlinear method defined on general non-uniform meshes is studied in Section 4. Finally, in Section 5, some numerical simulations are presented.

\section{The mesh equation}

Let us consider problem (2). Under the assumption that $c_{y}(y) \geq c_{0}^{\prime}, \forall y \in \mathbb{R}$, where $c_{0}^{\prime}$ is a positive constant, problem (2) has a unique solution as stated, for example, in [21]. If we solve it using a reasonable step-size and a standard numerical method, like centred finite-difference (CFD) approximations, results are very inaccurate (Figure 1). For a constant diffusion coefficient $D$, $A=1, B=-1, c(y)=0, b(y)=y$, problem (2) has the solution

$$
u(x)=\left\{\begin{array}{l}
\frac{1-e^{-D^{-1}(1 / 2-x)},}{1+e^{-D^{-1}(1 / 2-x)}} \quad x \in\left[0, \frac{1}{2}\right], \\
-\frac{1-e^{-D^{-1}(-1 / 2+x)}}{1+e^{-D^{-1}(-1 / 2+x)}}, \quad x \in\left(\frac{1}{2}, 1\right],
\end{array}\right.
$$

(see [21]).

To obtain accurate solutions, presenting no numerical dispersion, CFD should be used with a step-size satisfying

$$
h \leq \frac{2 D}{\|b\|_{\infty}}
$$

(see [21]). In Figure 2 we plot the numerical results obtained with a uniform grid with step-size $h=0.02$.

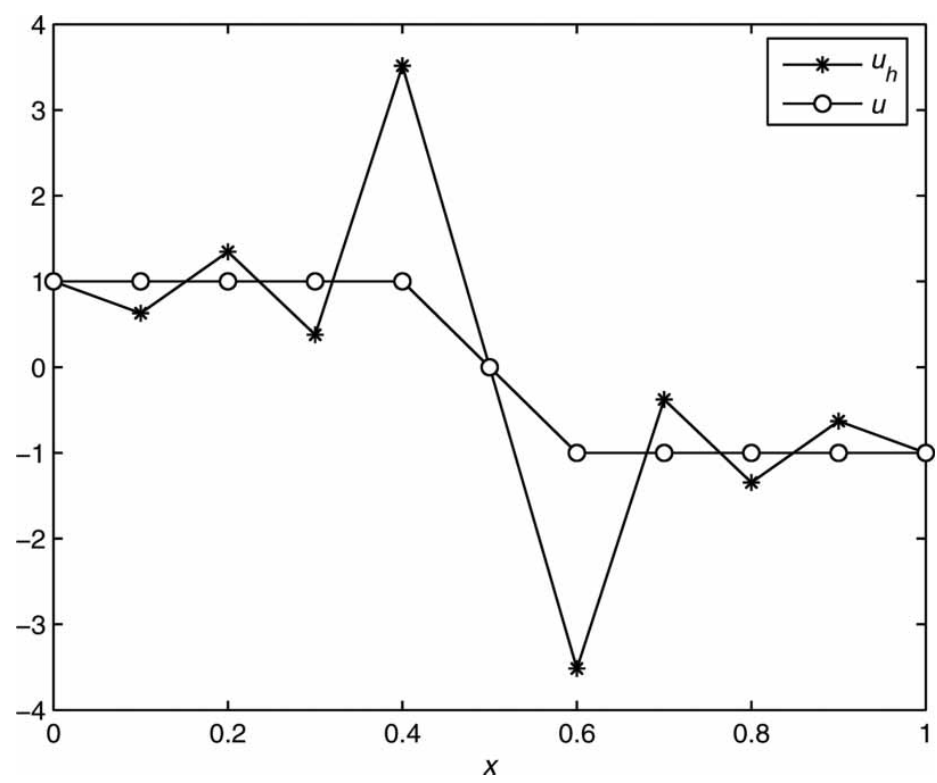

Figure 1. Exact $(u)$ and numerical $\left(u_{h}\right)$ solutions of Equation (2) for $A=1, B=-1, D=10^{-2}, b(y)=y, c(y)=0$ by using a uniform grid with step-size $h=0.1$. 


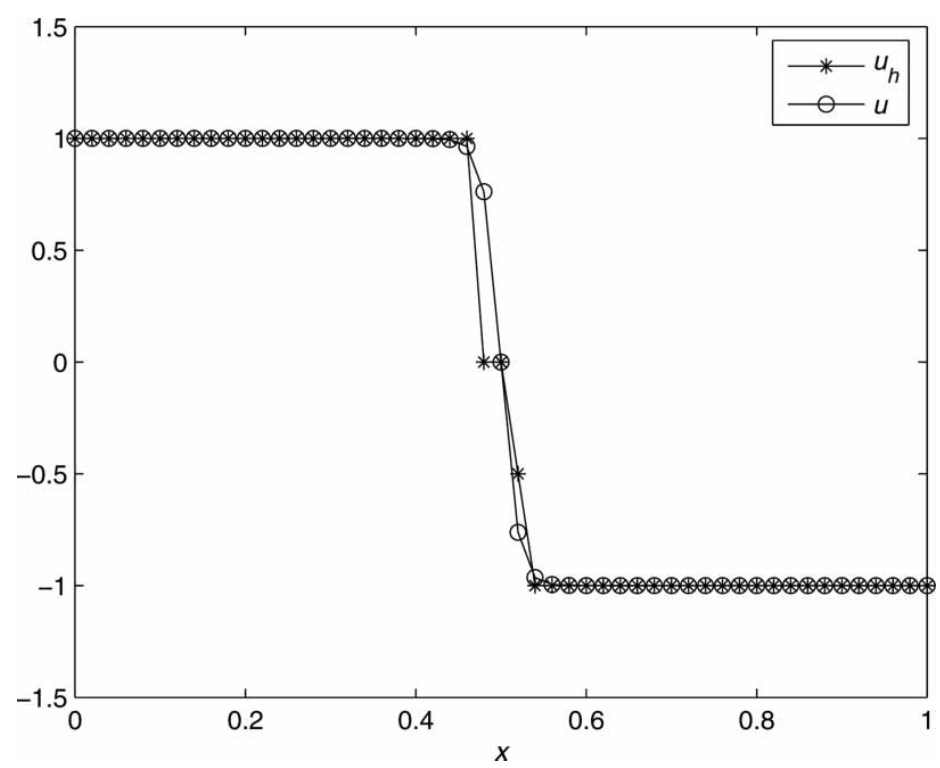

Figure 2. Exact $(u)$ and numerical $\left(u_{h}\right)$ solutions of (2) for $A=1, B=-1, D=10^{-2}, b(y)=y, c(y)=0$, by using a uniform grid with step-size $h=0.02$.

If upwind methods are used and $u$ changes sign at some $\bar{x} \in(0,1)$, which is not a priori known, we do not know where to switch the discretization of $u_{x}$ from forward to backward or from backward to forward. This approach would imply the use of upwind coupled with some 'layer predictor' to identify the turning point of $b$. In the case $u$ does not change sign it is well known that upwind method will produce solutions with a big amount of numerical dissipation.

Let $g:[0,1] \rightarrow[0,1]$ be such that $g(0)=0, g(1)=1$ and $g_{\xi}(\xi) \neq 0 \forall \xi \in(0,1)$. By $\bar{u}(\xi)$ we represent $u(g(\xi))$. In the following result we establish a characterization for $g$ such that $\left.u(x)=\bar{u}\left(g^{-1}(x)\right)\right)=(B-A) \xi+A$ is the solution of (2) at $x=g(\xi)$.

Proposition 1 Let $g:[0,1] \rightarrow[0,1]$ be such that $g(0)=0, g(1)=1, g_{\xi}(\xi) \neq 0, \forall \xi \in(0,1)$, and $\bar{u}(\xi)=(B-A) \xi+A$. If the mesh function $g$ satisfies the $B V P$

$$
\begin{gathered}
D(B-A) g_{\xi^{2}}+\bar{b}(\xi)(B-A) g_{\xi}^{2}+\bar{c}(\xi) g_{\xi}^{3}=0 \text { in }(0,1), \\
g(0)=0, g(1)=1,
\end{gathered}
$$

where $\bar{b}(\xi)=b((B-A) \xi+A), \bar{c}(\xi)=c((B-A) \xi+A)$, then $\bar{u}(\xi)=(B-A) \xi+A$ is the solution of Equation (2) at $x=g(\xi)$.

Proof Considering $x=g(\xi)$ in Equation (2) we obtain

$$
\begin{gathered}
-D \bar{u}_{\xi^{2}} \frac{1}{g_{\xi}^{2}}+\left(D \frac{g_{\xi^{2}}}{g_{\xi}^{3}} \bar{u}_{\xi}+\frac{\bar{b} \bar{u}_{\xi}}{g_{\xi}}+\bar{c}\right)=0 \quad \text { in }(0,1) \\
\bar{u}(0)=A, \quad \bar{u}(1)=B .
\end{gathered}
$$

Then taking $\bar{u}_{\xi^{2}}=0$, we conclude the proof.

We observe that if the coefficient functions $b$ and $c$ are continuous then Equation (5) has a unique solution [20]. 
We note that the procedure in Proposition 1 corresponds to selecting $g$ such that

$$
\frac{1}{g_{\xi}}=\frac{u_{x}}{B-A} \text {. }
$$

Defining the mesh density $d(\xi)$ by $1 / g_{\xi}$, this last equation corresponds to the natural choice of selecting $d(\xi)$ proportional to the solution gradient $u_{x}$.

To clarify the meaning of this change of variable, we consider in the next examples two particular cases.

Example 1 Let us consider $c=0, A=0, B=1$ and $b=$ constant. The solution of Equation (5) is given by

$$
g(\xi)=\frac{D}{b} \ln \left(1+\xi\left(\mathrm{e}^{b / D}-1\right)\right) .
$$

The mesh defined by Equation (7), $x_{i}=g\left(\xi_{i}\right), \xi_{i}=i / N, i=1, \ldots, N-1$, locates accurately the boundary layers, as can be seen from Table 1 .

Figures 3 and 4 show the plots of $g$, respectively, with $b>0$ and $b<0$ for $D=10^{-2}$.

For $b=1$ and $N=10$ the first node $x_{1}=1-O(D)$, where $O(D)$ represents a function of $D$ that converges to zero as $D$ goes to zero. We observe that all nodes are located in $\left[g\left(\xi_{1}\right), 1\right]$.

Table 1. The first node $x_{1}$ for $b=1$, $N=10$ and different values of $D$.

\begin{tabular}{lc}
\hline$D$ & $g\left(\xi_{1}\right)$ \\
\hline $10^{-2}$ & 0.9770 \\
$10^{-3}$ & 0.9977 \\
$10^{-4}$ & 0.9998 \\
\hline
\end{tabular}

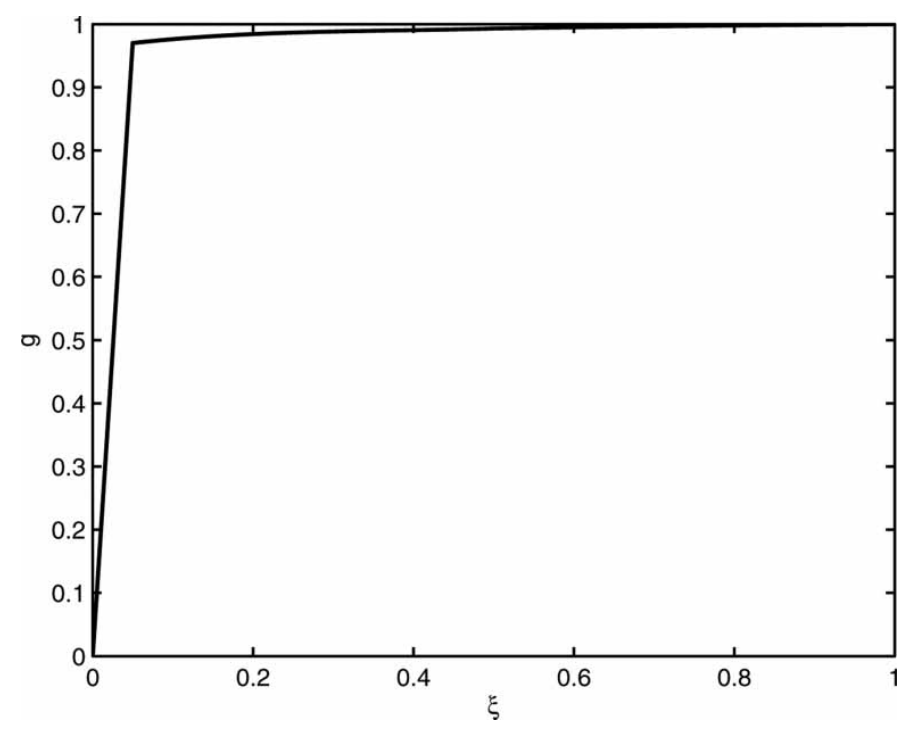

Figure 3. The plot of $g$ for $b=1$. 


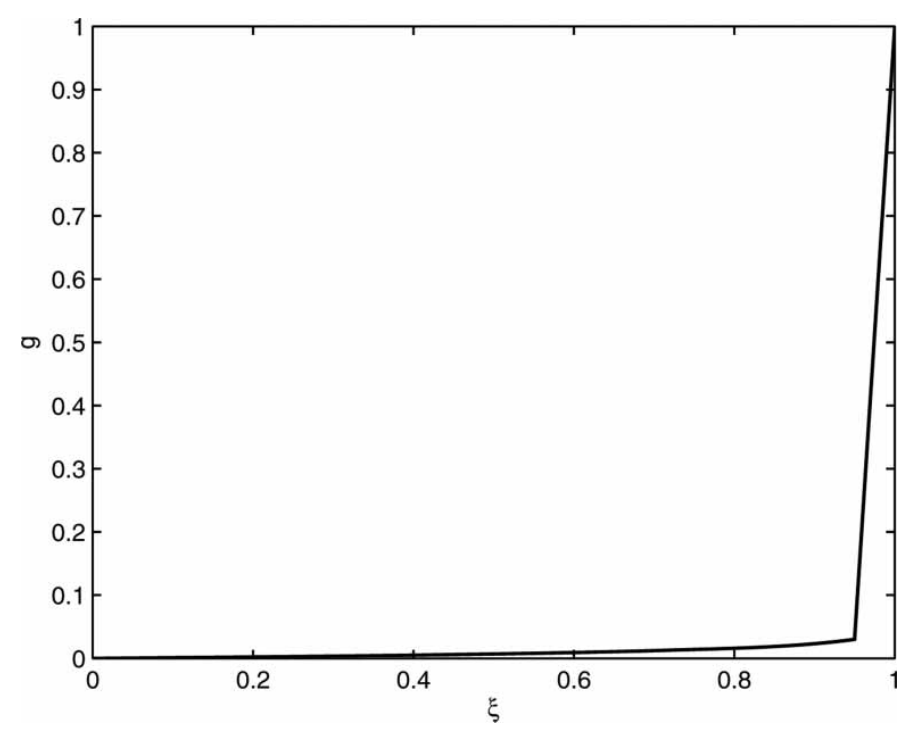

Figure 4. The plot of $g$ for $b=-1$.

Example 2 Let us assume that $b$ is non-constant with $b \neq 0$ in $(0,1)$, and $c=0$. From Equation (5) we obtain

$$
-\frac{g_{\xi^{2}}}{g_{\xi}^{2}}=\frac{\bar{b}(\xi)}{D}
$$

and then

$$
d(\xi)=\frac{1}{D} \int_{0}^{\xi} \bar{b}(s) \mathrm{d} s, \quad \xi \in(0,1] .
$$

If $b>0$ in $[0,1]$ then the mesh-density function $d$ is an increasing function of $\xi$, which means that a boundary layer exists at $x=1$. On the other hand, if $b<0$ in $[0,1]$, then $d$ is a decreasing function of $\xi$ and the boundary layer is located at $x=0$.

In the case that $\bar{b}$ changes sign in $(0,1)$ for some $\xi_{c}, d$ is not a monotone function. Let us consider, for instance, that $\bar{b}(\xi)>0$ in $\left[0, \xi_{c}\right)$ and $\bar{b}(\xi)<0$ in $\left(\xi_{c}, 1\right]$. As before, we conclude that $d$ is an increasing function in $\left[0, \xi_{c}\right)$ and a decreasing function in $\left(\xi_{c}, 1\right]$, which indicates the existence of an internal layer at $\xi=\xi_{c}$. If we consider $\bar{b}(\xi)<0$ in $\left[0, \xi_{c}\right)$ and $\bar{b}(\xi)>0$ in $\left(\xi_{c}, 1\right]$, we expect two boundary layers located at $x=0$ and $x=1$.

In Examples 1 and 2, an interpretation of the mesh-density function in some particular cases was given. In a general case, mesh Equation (5) cannot be solved exactly. Next section is devoted to the study of numerical discretizations of Equation (5).

We point out that the approach introduced in this paper for one-dimensional domain can be generalized to domains of type $\Omega=\prod_{i=1}^{n}\left(a_{i}, b_{i}\right)$. In fact, considering in Equation (1) $x=\left(x_{1}, \ldots, x_{n}\right)$ with $x_{i}=g_{i}\left(\xi_{i}\right), g_{i}:[0,1] \rightarrow\left[a_{i}, b_{i}\right], i=1, \ldots, n$, problem (1) is equivalent to a modified system of differential equations in the dependent variables $g_{1}\left(\xi_{1}\right), \ldots, g_{n}\left(\xi_{n}\right)$. 


\section{The discrete mesh equation}

Let $v_{h}$ and $v_{k}$ be mesh functions defined in the meshes $\left\{x_{\ell}, \ell=0, \ldots, N\right\}$ and $\left\{\xi_{\ell}, \ell=0, \ldots, N\right\}$, respectively, with

$$
x_{0}=\xi_{0}=0, \quad x_{N}=\xi_{N}=1, \quad x_{\ell+1}-x_{\ell}=h_{\ell+1}, \quad \ell=0, \ldots, N-1, \quad k=\frac{1}{N}
$$

By $D_{c, x} v_{h}$ we represent the first-order centred difference

$$
D_{c, x} v_{h}\left(x_{i}\right)=\frac{v_{h}\left(x_{i+1}\right)-v_{h}\left(x_{i-1}\right)}{h_{i+1}+h_{i}} .
$$

The centred difference $D_{c, \xi} v_{k}$ is defined analogously to Equation (10) in the uniform grid $\left\{\xi_{\ell}\right\}$. The centred difference operator $D_{2, x}$ is given by

$$
D_{2, x} v_{h}\left(x_{i}\right)=\frac{h_{i} v_{h}\left(x_{i+1}\right)-v_{h}\left(x_{i}\right)\left(h_{i+1}+h_{i}\right)+h_{i+1} v_{h}\left(x_{i-1}\right)}{h_{i+1} h_{i}\left(h_{i+1}+h_{i}\right) / 2} .
$$

$D_{2, \xi} v_{k}\left(\xi_{i}\right)$ represents the standard centred difference operator defined in a uniform mesh of stepsize $k$. Finally, by $D_{-x}$ and $D_{-\xi}$ we represent the backward operators for mesh functions with values in the meshes $\left\{x_{\ell}\right\}$ and $\left\{\xi_{\ell}\right\}$, respectively.

We consider the following discretizations of the mesh Equation (5):

(1) If $B>A$

$$
D(B-A) D_{2, \xi} g_{k}\left(\xi_{i}\right)+D_{-\xi} g_{k}\left(\xi_{i+1}\right) D_{-\xi} g_{k}\left(\xi_{i}\right)\left((B-A) \bar{b}_{i}+\bar{c}_{i} D_{-\xi} g_{k}\left(\xi_{i+1}\right)\right)=0,
$$

for $i=1, \ldots, N-1$, with $g_{k}\left(\xi_{0}\right)=0, g_{k}\left(\xi_{N}\right)=1$;

(2) If $B<A$

$$
D(B-A) D_{2, \xi} g_{k}\left(\xi_{i}\right)+D_{-\xi} g_{k}\left(\xi_{i+1}\right) D_{-\xi} g_{k}\left(\xi_{i}\right)\left((B-A) \bar{b}_{i}+\bar{c}_{i} D_{-\xi} g_{k}\left(\xi_{i}\right)\right)=0,
$$

for $i=1, \ldots, N-1$, with $g_{k}\left(\xi_{0}\right)=0, g_{k}\left(\xi_{N}\right)=1$.

In the following we consider $B>A$. For $B<A$ similar results are obtained.

Proposition 2 Let us assume that $B>A$ and that the spatial mesh $\left\{x_{i}\right\}=\left\{g_{k}\left(\xi_{i}\right)\right\}$ is computed using the finite-difference method (12). Then $u_{h}$ given by

$$
u_{h}\left(x_{0}\right)=A, \quad u_{h}\left(x_{N}\right)=B, \quad u_{h}\left(x_{i}\right)=\bar{u}\left(\xi_{i}\right)=(B-A) \xi_{i}+A, \quad i=1, \ldots, N-1,
$$

satisfies, for $i=1, \ldots, N-1$,

$$
-D D_{2, x} u_{h}\left(x_{i}\right)+\bar{b}_{i} D_{c, x} u_{h}\left(x_{i}\right)+\bar{c}_{i}\left(1+\frac{h_{i+1}-h_{i}}{h_{i+1}+h_{i}}\right)=0,
$$

where $h_{\ell}=x_{\ell}-x_{\ell-1}=g_{k}\left(\xi_{\ell}\right)-g_{k}\left(\xi_{\ell-1}\right), \ell=1, \ldots, N$. 
Proof The mesh $\left\{g_{k}\left(\xi_{\ell}\right)\right\}$ is defined by Equation (12), which is equivalent to

$$
\begin{aligned}
2 k(B & -A) D \frac{g_{k}\left(\xi_{i+1}\right)-2 g_{k}\left(\xi_{i}\right)+g_{k}\left(\xi_{i-1}\right)}{\left(g_{k}\left(\xi_{i+1}\right)-g_{k}\left(\xi_{i}\right)\right)\left(g_{k}\left(\xi_{i}\right)-g_{k}\left(\xi_{i-1}\right)\right)}+2 k(B-A) \bar{b}_{i} \\
& +2 \bar{c}_{i}\left(g_{k}\left(\xi_{i+1}\right)-g_{k}\left(\xi_{i}\right)\right)=0 .
\end{aligned}
$$

Noting that

$$
u_{h}\left(x_{i+1}\right)-u_{h}\left(x_{i-1}\right)=(B-A)\left(\xi_{i+1}-\xi_{i-1}\right)=2 k(B-A)
$$

and also

$$
2 k D_{c, \xi} g_{k}\left(\xi_{i}\right)=h_{i}+h_{i+1}, \quad k^{2} D_{2, \xi} g_{k}\left(\xi_{i}\right)=h_{i+1}-h_{i}, \quad k D_{-\xi} g_{k}\left(\xi_{i+1}\right)=h_{i+1},
$$

we conclude that $u_{h}$ satisfies

$$
D \frac{h_{i+1}-h_{i}}{h_{i} h_{i+1}}\left(u_{h}\left(x_{i+1}\right)-u_{h}\left(x_{i-1}\right)\right)+\bar{b}_{i}\left(u_{h}\left(x_{i+1}\right)-u_{h}\left(x_{i-1}\right)\right)+2 \bar{c}_{i} h_{i+1}=0 .
$$

Finally, as $u_{h}\left(x_{i+1}\right)-2 u_{h}\left(x_{i}\right)+u_{h}\left(x_{i-1}\right)=0$, we establish from Equation (17) that

$$
\begin{aligned}
& D\left(-\left(u_{h}\left(x_{i+1}\right)-2 u_{h}\left(x_{i}\right)+u_{h}\left(x_{i-1}\right)\right) \frac{h_{i+1}-h_{i}}{h_{i} h_{i+1}}+\frac{h_{i+1}-h_{i}}{h_{i} h_{i+1}}\left(u_{h}\left(x_{i+1}\right)-u_{h}\left(x_{i-1}\right)\right)\right) \\
& \quad+\bar{b}_{i}\left(u_{h}\left(x_{i+1}\right)-u_{h}\left(x_{i-1}\right)\right)+2 \bar{c}_{i} h_{i+1}=0,
\end{aligned}
$$

which allows us to conclude that $u_{h}$ satisfies Equation (15).

We proved that the approximation $u_{h}\left(x_{i}\right)$ of $u\left(x_{i}\right)$ defined by $u_{h}\left(x_{i}\right)=(B-A) \xi_{i}+A$ for $x_{i}$ such that $x_{i}=g(\xi)$ is solution of the nonlinear difference Equation (15) computed in the mesh given by Equation (12). Following this result we solve mesh Equation (12) obtaining $g_{k}\left(\xi_{i}\right)$, that is, the mesh point $x_{i}$ where the numerical approximation for the solution is evaluated. The solution of the mesh equation furnishes immediately an approximated solution for problem (2).

At this point it could be asked what is the advantage of replacing the direct solution of problem (2) by the solution of mesh Equation (5) using Equation (12), as stated in Proposition 2. Two main points can be argued:

- the numerical solutions obtained using Equation (12) are convergent, positive, and nonoscillatory, as we will prove in what follows.

- the smoothness of the coefficient functions is irrelevant in the procedure, which is not the case when Equation (2) is solved.

THEOREM 1 Let c be positive and $g_{k}$ be defined by Equation (12). Then $g_{k}\left(\xi_{i}\right) \geq 0, i=0, \ldots, N$, and

$$
g_{k}\left(\xi_{i+1}\right) \geq g_{k}\left(\xi_{i}\right), \quad i=1, \ldots, N-1 .
$$

Proof Let $G: \mathbb{R}^{N-1} \rightarrow \mathbb{R}^{N-1}, G=\left(G_{1}, \ldots, G_{N-1}\right)$, be the nonlinear mapping defined by

$$
G_{i}\left(y_{1}, \ldots, y_{N-1}\right)=(A-B) D \frac{y_{i+1}-2 y_{i}+y_{i-1}}{\left(y_{i+1}-y_{i}\right)\left(y_{i}-y_{i-1}\right)}+(A-B) \bar{b}_{i}+\bar{c}_{i} \frac{y_{i}-y_{i+1}}{k}
$$

for $i=1, \ldots, N-1$, and for $y=\left(y_{1}, \ldots, y_{N-1}\right) \in \mathbb{R}^{N-1}$.

Then Equation (12) is equivalent to $G\left(g_{k}\left(\xi_{1}\right), \ldots, g_{k}\left(\xi_{N-1}\right)\right)=0$. To prove that $g_{k}$ is nonnegative, it is enough to show that $G$ is an inverse monotone function [5], which means that, if $G\left(g_{k}\right) \geq 0$ then $g_{k} \geq 0$. 
If the Jacobian matrix of $G, J G$, is inverse monotone, by using the Mean Value Theorem for each coordinate function $G_{i}$ of $G$, it is easily proved that $G$ is inverse monotone. We begin by proving that $J G$ is inverse monotone. The components of $J G$ at $\left(g_{k}\left(\xi_{1}\right), \ldots, g_{k}\left(\xi_{N-1}\right)\right)$ are defined by

$$
J G_{i j}=\left\{\begin{array}{l}
\frac{D(A-B)}{k^{2} D_{-\xi} g_{k}\left(\xi_{i+1}\right)^{2}}-\frac{\bar{c}_{i}}{k}, \quad j=i+1 \\
D(B-A) \frac{D_{-\xi} g_{k}\left(\xi_{i+1}\right)^{2}+D_{-\xi} g_{k}\left(\xi_{i}\right)^{2}}{k^{2} D_{-\xi} g_{k}\left(\xi_{i+1}\right)^{2} D_{-\xi} g_{k}\left(\xi_{i}\right)^{2}}+\frac{\bar{c}_{i}}{k}, \quad j=i \\
\frac{D(A-B)}{k^{2} D_{-\xi} g_{k}\left(\xi_{i}\right)^{2}}, \quad j=i-1 \\
0, \quad \text { in other cases. }
\end{array}\right.
$$

The matrix $J G$ is a tridiagonal matrix whose diagonal entries are positive and whose non-diagonal entries are non-positive. Then every $i \in\{1, \ldots, N-1\}$ is connected with every $j \in\{1, \ldots, N-$ $1\}$, which means that $J G$ is irreducible. Considering that $J G$ is diagonally dominant for all $k$, we conclude that $J G$ is a $M$-matrix and consequently $(J G)^{-1} \geq 0$ (see [15]). As $J G$ is inverse monotone, $G$ is an inverse monotone operator and, consequently, $G\left(g_{k}\right) \geq 0$ implies $g_{k} \geq 0$.

We prove in what follows that $g_{k}$ satisfies Equation (19). Let $v_{k}\left(\xi_{i}\right)=g_{k}\left(\xi_{i}\right)-g_{k}\left(\xi_{i-1}\right), i=$ $1, \ldots, N-1$. From Equation (12) we have

$$
D(B-A) \frac{v_{k}\left(\xi_{i+1}\right)-v_{k}\left(\xi_{i}\right)}{v_{k}\left(\xi_{i}\right) v_{k}\left(\xi_{i+1}\right)}+(B-A) \bar{b}_{i}+\bar{c}_{i} \frac{v_{k}\left(\xi_{i+1}\right)}{k}=0, \quad i=1, \ldots, N-1 .
$$

If the nonlinear mapping $G_{d}: \mathbb{R}^{N-1} \rightarrow \mathbb{R}^{N-1}, G_{d}=\left(G_{d, 1}, \ldots, G_{d, N-1}\right)$ is defined by

$$
G_{d, i}\left(y_{1}, \ldots, y_{N-1}\right)=D(A-B) \frac{y_{i+1}-y_{i}}{y_{i} y_{i+1}}+(A-B) \bar{b}_{i}-\bar{c}_{i} \frac{y_{i+1}}{k},
$$

for $i=1, \ldots, N-1,\left(y_{1}, \ldots, y_{N-1}\right) \in \mathbb{R}^{N-1}$, then Equation (21) is equivalent to $G_{d}\left(v_{k}\left(\xi_{1}\right), \ldots\right.$, $\left.v_{k}\left(\xi_{N-1}\right)\right)=0$. To prove that $g_{k}$ satisfies Equation (19), we prove that $v_{k} \geq 0$. It is enough to show that $G_{d}$ is an inverse monotone function. Following the proof of the inverse monotony of $G$ we study the properties of $J G_{d}$ at $\left(g_{k}\left(\xi_{1}\right), \ldots, g_{k}\left(\xi_{N-1}\right)\right)$ defined by

$$
J G_{d_{i j}}=\left\{\begin{array}{l}
\frac{D(B-A)}{v_{k}\left(\xi_{i}\right)^{2}}, \quad j=i \\
-\frac{D(B-A)}{v_{k}\left(\xi_{i+1}\right)^{2}}-\frac{\bar{c}_{i}}{k}, \quad j=i+1, \\
0, \quad \text { in other cases. }
\end{array}\right.
$$

The matrix $J G_{d}$ is an $M$-matrix and then $G_{d}$ is inverse monotone. Therefore $G_{d}\left(v_{k}\right) \geq 0$ implies $v_{k} \geq 0$.

\section{Convergence properties}

In Proposition 2 it was established that the numerical solution $u_{h}$ defined by Equation (14) can be computed using the finite-difference scheme (15) with the mesh (12) (or (13)). For general 
non-uniform meshes, the finite-difference scheme

$$
F_{h}\left(u_{h}\right)=0
$$

with

$$
\begin{aligned}
F_{h}\left(u_{h}\left(x_{i}\right)\right)= & -D D_{2, x} u_{h}\left(x_{i}\right)+b\left(u_{h}\left(x_{i}\right)\right) D_{c, x} u_{h}\left(x_{i}\right) \\
& +c\left(u_{h}\left(x_{i}\right)\right)\left(1+\frac{h_{i+1}-h_{i}}{h_{i}+h_{i+1}}\right), \quad i=1, \ldots, N-1,
\end{aligned}
$$

where $u_{h}\left(x_{0}\right)=A, u_{h}\left(x_{N}\right)=B$ and $h=\left(h_{1}, \ldots, h_{N}\right)$, is inconsistent with respect to the $L_{\infty}$ norm. In fact the truncation error $T_{h}$ is such that $\left\|T_{h}\right\|_{\infty}$ does not converge to zero when $h \rightarrow 0$. However, we establish in what follows that method (23) is convergent, provided that the nonuniform $\left\{x_{i}\right\}$ is defined by Equation (12). We start by noting that in the computational grid $\left\{x_{i}\right\}$, $x_{i}=g_{k}\left(\xi_{i}\right), i=0, \ldots, N$, we have

$$
\begin{aligned}
u\left(x_{i}\right)-u_{h}\left(x_{i}\right) & =u\left(x_{i}\right)-\bar{u}\left(\xi_{i}\right) \\
& =u\left(x_{i}\right)-u\left(g\left(\xi_{i}\right)\right) \\
& =u_{x}\left(\eta_{i}\right)\left(g_{k}\left(\xi_{i}\right)-g\left(\xi_{i}\right)\right),
\end{aligned}
$$

where $\eta_{i}=\theta g_{k}\left(\xi_{i}\right)+(1-\theta) g\left(\xi_{i}\right), \theta \in[0,1]$. An estimate to $\left\|R_{h} u-u_{h}\right\|_{\infty}$, where $R_{h}$ denotes the restriction operator to the grid $\left\{x_{i}\right\}$, is obtained by estimating $\left\|g_{k}-R_{k} g\right\|_{\infty}$, where $R_{k}$ denotes the restriction operator to the grid $\left\{\xi_{i}\right\}$. We recall that $g_{k}\left(\xi_{0}\right)=0, g_{k}\left(\xi_{N}\right)=1$, and $g_{k}\left(\xi_{i}\right), i=$ $1, \ldots, N-1$, is a solution of $G\left(g_{k}\right)=0$, where $G$ is defined in the proof of Theorem 1 .

It has been proved that $J G\left(R_{k} g\right)$ is an $M$-matrix under the assumption $c \geq 0$. In this case it can be shown that $\left\|J G\left(R_{k} g\right)^{-1}\right\|_{\infty} \leq C$, where $C$ represents a positive constant. In fact, as $J G\left(R_{k} g\right)$ is an $M$-matrix and

$$
\left(J G\left(R_{k} g\right) R_{k} \hat{g}\right)_{i} \geq 1, \quad i=1, \ldots, N-1
$$

for a certain $\hat{g}$ depending on $g$, we have

$$
\left\|J G\left(R_{k} g\right)^{-1}\right\|_{\infty} \leq\left\|R_{k} \hat{g}\right\|_{\infty}
$$

For instance, if $g^{\prime \prime}<0$ then we can choose $\hat{g}=-g / g_{\min }^{\prime} c_{0}$. Otherwise if $g_{\max }^{\prime \prime}>0$ then $\hat{g}=$ $-g / g_{\min }^{\prime} c_{0} \eta$, provided that $\eta$ is such that $-D(B-A) \eta g_{\max }^{\prime \prime}+1 / \eta \geq 1$.

Furthermore, we have

$$
\left\|J G\left(\tilde{g}_{k}\right)-J G\left(\breve{g}_{k}\right)\right\|_{\infty} \leq L\left\|\tilde{g}_{k}-\breve{g}_{k}\right\|_{\infty}, \quad \tilde{g}_{k}, \breve{g}_{k} \in B_{\rho}\left(R_{k} g\right) \cap D_{G},
$$

where $L$ denotes a positive constant, $D_{G}$ represents the domain of $G$ and $B_{\rho}\left(R_{k} g\right)$ denotes the open ball centred at $R_{k} g$ and radius $\rho$. For instance, Equation (27) holds with $\rho$ such that $\rho<\min _{i} g\left(\xi_{i}\right)-g\left(\xi_{i-1}\right)$.

Using Lemma 3.5 and Theorem 3.6 of [13] we conclude, from Equations (26), (27) and from the consistency of the discretization (12) with (5), the following:

$$
\left\|R_{h} u-u_{h}\right\|_{\infty} \longrightarrow 0, \quad k \longrightarrow 0
$$

that is, method (23) is convergent. 


\section{Numerical results}

In this section we illustrate the performance of the procedure.

Example 3 Let us consider the steady convection-diffusion-reaction BVP

$$
\begin{gathered}
-D u_{x^{2}}+(u-0.5) u_{x}+p u^{2}=0 \quad \text { in }(0,1), \\
u(0)=1, \quad u(1)=0,
\end{gathered}
$$

where $p$ is a positive parameter and $D=10^{-2}$.

In Figure 5 we plot three solutions:

(1) the reference solution obtained with a CFD scheme defined on a uniform mesh with step-size $h=10^{-2}$ (circles);

(2) the lifting solution computed with $k=10^{-1}$ (squares);

(3) an upwind solution (stars) computed with

$$
-D D_{2, x} u_{h}\left(x_{i}\right)+u_{h}\left(x_{i}\right) D_{-x} u_{h}\left(x_{i}\right)-0.5 D_{x} u_{h}\left(x_{i}\right)=0, \quad i=1, \ldots, N-1,
$$

with $u_{h}\left(x_{0}\right)=1, u_{h}\left(x_{N}\right)=0$, for $h=10^{-1}$.

As proved previously, a positive and non-oscillatory lifting solution is obtained with no restriction on $k$. We observe that the proposed lifting solution presents the most accurate result even when a coarse mesh is employed.

In Figure 6 an upwind solution computed with $h=10^{-2}$ (stars) is compared with the lifting solution defined with $k=10^{-1}$ (squares). This solution still exhibits numerical dissipation.

Figure 7 illustrates the behaviour of the lifting solutions of Equation (29) for increasing values of $p$. The computations have been made using mesh points plotted in Figure 8. The internal

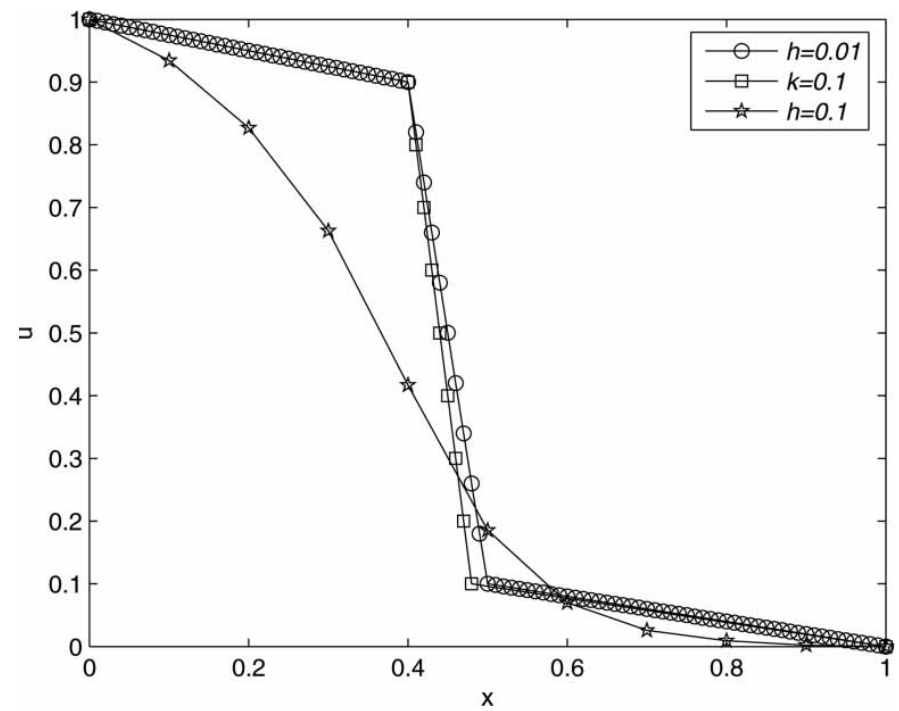

Figure 5. Numerical solutions of Equation (29) $\left(p=0.01, D=10^{-2}\right)$ obtained with the upwind Scheme (30) for $h=10^{-1}$ (stars), by using the mesh solution defined by Equation (13) with $k=10^{-1}$ (squares) and the reference solution (circles). 


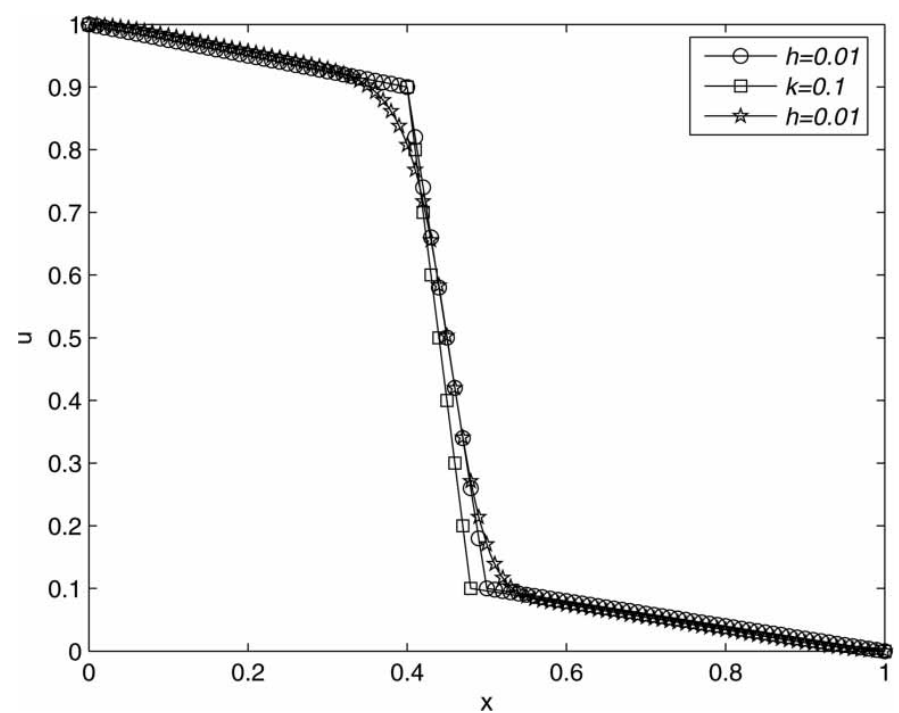

Figure 6. Numerical solution of Equation (29) $\left(p=0.01, D=10^{-2}\right)$ obtained with the upwind Scheme (30)(stars), the lifting solution for $k=10^{-1}$ (squares) and the reference solution (circles).

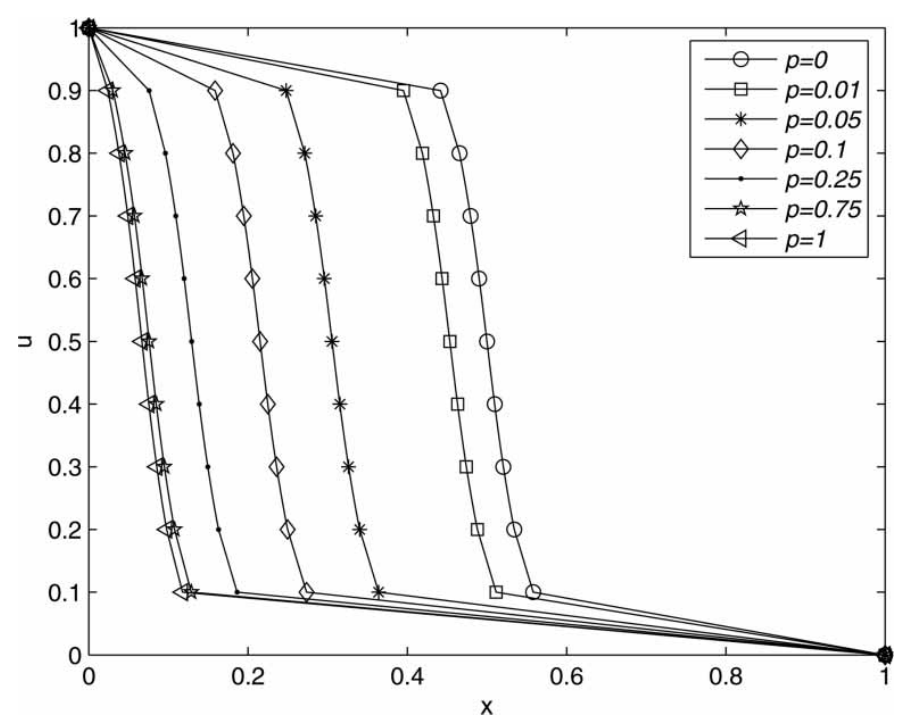

Figure 7. Lifting solutions of Equation (29) with $D=10^{-2}, k=10^{-1}$ for different values of $p$.

boundary layer is correctly located moving to the left as $p$ approaches 1 . We note that for each problem the numerical $x$-grid is the projection in the vertical axe of the grid points.

Example 4 Let us consider the steady convection-diffusion BVP

$$
\begin{gathered}
-D u_{x^{2}}+(u-a) u_{x}=0 \quad \text { in }(0,1), \\
u(0)=0, \quad u(1)=1,
\end{gathered}
$$

with $a \in[0,1)$ and $D=10^{-2}$. For $a=0$, we have Burger's equation. 


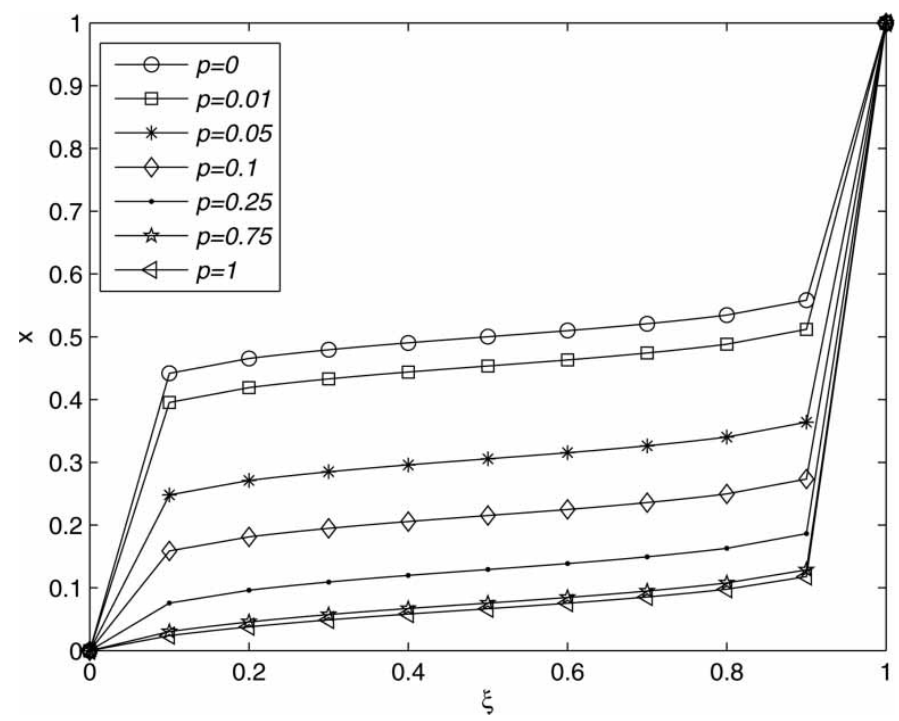

Figure 8. Numerical grids defined by Equation (13) for the BVP (29) obtained with $k=10^{-1}$.

In Figure 9 we plot the reference solution (circles) obtained with a CFD scheme defined on a uniform mesh with stepsize $h=10^{-2}$ and the lifting solution (squares) obtained with $k=10^{-1}$.

Figure 10 shows the behaviour of the lifting solutions for different values of $a$. We can observe for $a=0$ and $a=0.75$ a boundary layer, respectively, located at $x=1$ and $x=0$. For $a=0.25$ and $a=0.5$ the solution has two internal layers. The computations have been made using mesh points plotted in Figure 11.

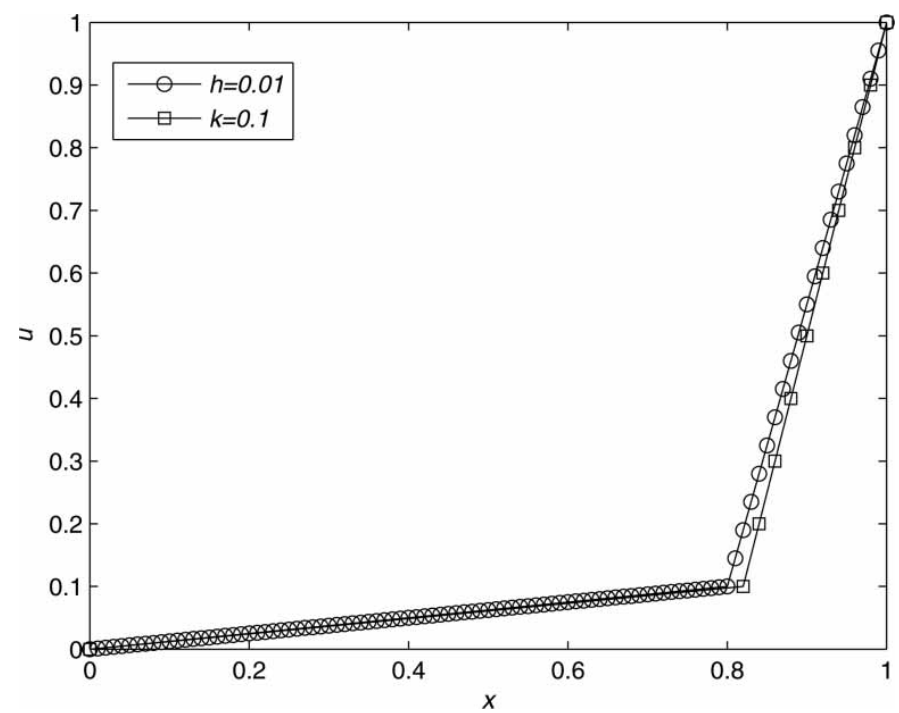

Figure 9. The reference solution for Equation (31) $\left(a=0, D=10^{-2}\right)$ (circles) and the lifting solution for $k=10^{-1}$ (squares). 


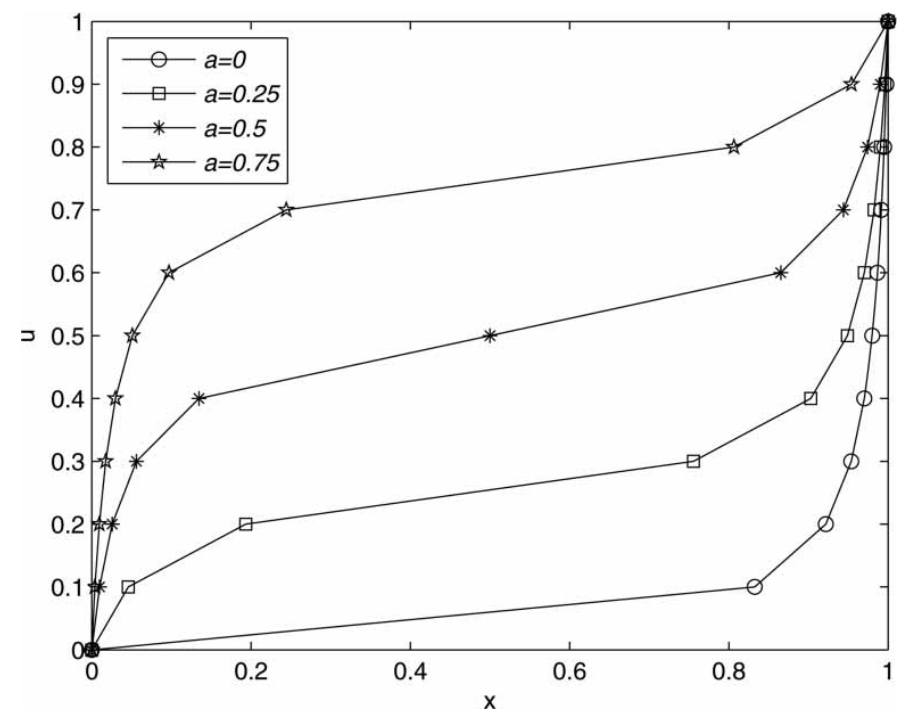

Figure 10. Lifting solutions for Equation (31) with $D=10^{-2}, k=10^{-1}$ for $a=0,0.25,0.5,0.75$.

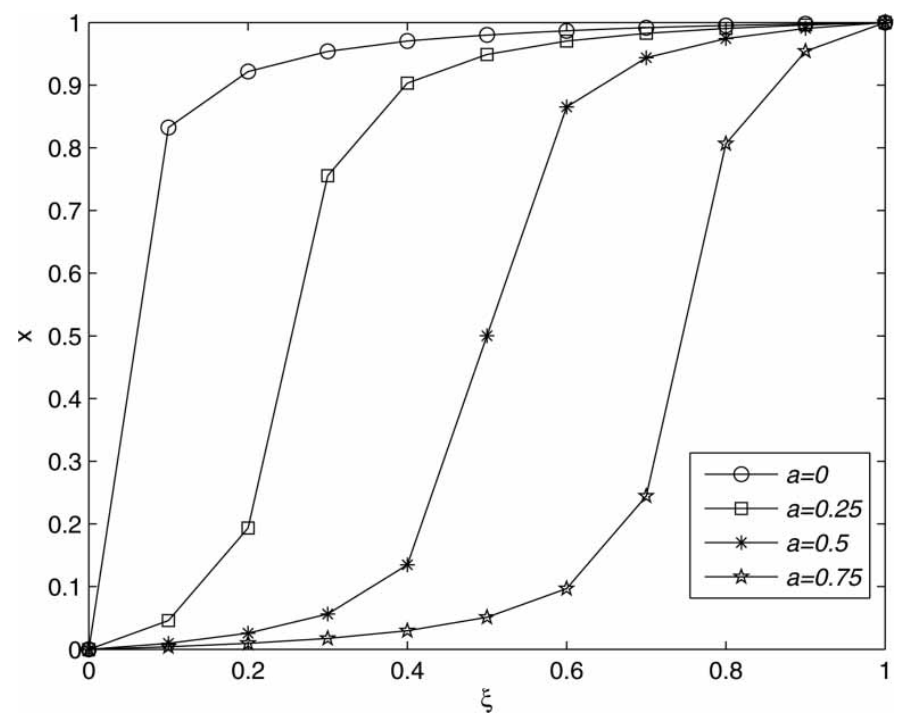

Figure 11. Numerical grids defined by Equation (12) for the BVP (31) obtained with $k=10^{-1}$.

\section{Conclusions}

A new method to numerically solve BVPs of type (2) is presented in this paper. The difficulties of the numerical solution of such BVPs are associated with the localization of layers, boundary layers and/or internal layers, which are not a priori known. Then the computation of a numerical approximation for the solution (2) usually involves the following three steps:

- (i) the localization of the layer(s) by solving the BVP with some 'predictor' method,

- (ii) the definition of the non-uniform grid,

- (iii) the computation of the numerical solution defined on the non-uniform grid. 
The numerical method introduced in this paper has only one step: step (ii). In fact we only need to compute a mesh function $g$ with the 'natural' property that the mesh density, represented by $1 / g_{\xi}$, is proportional to $u_{x}$, where $u$ is the solution of the BVP. Once we compute $g_{k}\left(\xi_{\ell}\right)-$ an approximation of $g$ at a uniform grid $\xi_{\ell}$ - the numerical approximation $u_{h}\left(x_{\ell}\right)$ of $u\left(x_{\ell}\right)$ for $x_{\ell}=g_{k}\left(\xi_{\ell}\right)$ is immediately given by $u_{h}\left(x_{\ell}\right)=(B-A) \xi_{\ell}+A$.

As our approach avoids steps (i) and (iii), usually followed by methods defined on non-uniform grids, our method requires less memory.

We established in Proposition 2 that the numerical solution obtained with our procedure can be computed using the non-standard, nonlinear, finite-difference scheme (15), defined on a nonuniform mesh given by Equation (12) (or (13)). As this scheme is first-order convergent we conclude that the approach introduced leads to a first-order approximation for the solution of BVP (2). We remark that CFD schemes and upwind schemes are second and first-order convergent on non-uniform grids, respectively.

It is well known that centred schemes are second-order consistent, but they produce oscillatory solutions when a reasonable number of mesh points are used (Figures 1 and 2). Upwind schemes introduce a big amount of numerical dissipation and to decrease such dissipation the numerical mesh should be refined (Figures 5 and 6). The proposed methodology, while using coarser grids, represents in a certain sense a qualitative compromise between upwind methods and centred methods: it is unconditionally non-oscillatory as upwind schemes, and it exhibits non-diffusive profiles as centred methods.

\section{Acknowledgements}

The authors gratefully acknowledge the support of this work by the Centro de Matemática da Universidade de Coimbra. The authors also thank the anonymous referees for their helpful comments. This work was supported by CMUC and project PTDC/MAT/74548/2006.

\section{References}

[1] J. Bea and Y. Bachmat, Introduction to Modeling of Transport Phenomena in Porous Media, Kluwer Academic Publishers, London, 1991.

[2] R. Bürgera, A. Coronelb, and M. Sepúlveda, On an upwind difference scheme for strongly degenerate parabolic equations modelling the settling of suspensions in centrifuges and non-cylindrical vessels, Appl. Numer. Math. 56 (2006), pp. 1397-1417.

[3] G. Chavent and J. Jaffé, Mathematical Models and Finite Elements for Reservoir Simulation, North-Holland, Amsterdam, 1986.

[4] Z. Chen and G. Ji, Sharp $L^{1}$ a posteriori error analysis for nonlinear convection-diffusion problems, Math. Comp. 75 (2006), pp. 43-71.

[5] L. Debnath, Nonlinear Partial Differential Equations for Scientists and Engineers, Birkhäuser, Boston, 1997.

[6] R.E. Ewing, The mathematics of reservoir simulation. Frontiers in Applied Mathematics, 1, Society for Industrial and Applied Mathematics (SIAM), Philadelphia, PA, 1983.

[7] R.E. Ewing and H. Wang, A summary of numerical methods for time-dependent advection-dominated partial differential equations, J. Comput. Appl. Math. 128 (2001), pp. 423-455.

[8] K.B. Fabinda, A linearization of a backward Euler scheme for a class of degenerate nonlinear advection-diffusion equations, Nonlinear Anal. 63 (2005), pp. 1097-1106.

[9] P.A. Farrell, E. O’Riordan, and I. Shishkin, A class of singularly perturbed semilinear differential equations with interior layers, Math. Comp. 74 (2005), pp. 1759-1776.

[10] A.C. Galeão, R.C. Almeida, S.M.C. Malta, and A.F.D. Loula, Finite element analysis of convection dominated reaction-diffusion problems, Appl. Numer. Math. 48 (2005), pp. 205-222.

[11] M. Javidi, Spectral collocation method for the solution of the generalized Burger-Fisher equation, Appl. Math. Comp. 174 (2006), pp. 345-352.

[12] K.H. Karlsen, N.J. Risebro, and J.D. Towers, On a nonlinear degenerate parabolic transportdiffusion equation with a discontinuous coefficient, Electron. J. Differential Equations 93 (2002), pp. 1-23.

[13] H.B. Keller, Approximation methods for nonlinear problems with application to two-point boundary value problems, Math. Comp. 29 (1975), pp. 464-474.

[14] H.V. Kojouharov and B.M Chen-Charpentier, Nonstandard Eulerian-Lagrangian methods for multidimensional reactive transport problems, Appl. Numer. Math. 49 (2004), pp. 225-243. 
[15] W. Hackbusch, Elliptic Differential Theory and Numerical Treatment, Springer-Verlag, Berlin, 1992.

[16] T. Linß, Sufficient conditions for uniform convergence on layer-adapted meshes for one-dimensional reactiondiffusion problems, Numer. Algorithms 40 (2005), pp. 23-32.

[17] T. Linß, On a set of singularly perturbed convection-diffusion equations, J. Comput. Appl. Math. 180 (2005), pp. $173-179$.

[18] C. Liu, Exact analytical solutions for nonlinear reaction-diffusion equations, Chaos Solitons Fractals 18 (2003), pp. 97-105.

[19] J.D. Murray, Mathematical Biology, Springer, Berlin, 1993.

[20] R.E. O’Malley, Singular Perturbation Methods for Ordinary Differential Equations, Springer, Berlin, 1991.

[21] H.G. Roos, M. Stynes, and L. Tobiska, Numerical Methods for Singularly Perturbed Differential Equations, SpringerVerlag, Berlin, 1996

[22] R. Sinha and J. Geiser, Error estimates for finite-volume-element methods for convection-diffusion-reaction equations, Appl. Numer. Math. 57 (2007), pp. 59-72. 